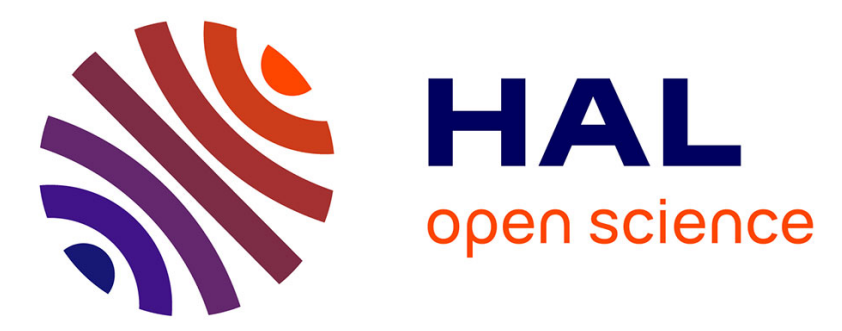

\title{
Instabilité et bifurcation du soufflage de membranes hyperélastiques
}

Nicolas Chevaugeon, Gilles Marckmann, Erwan Verron, Bernard Peseux

\section{To cite this version:}

Nicolas Chevaugeon, Gilles Marckmann, Erwan Verron, Bernard Peseux. Instabilité et bifurcation du soufflage de membranes hyperélastiques. Revue Européenne des Éléments Finis, 2002, 11 (2-4), pp.479 - 492. 10.3166/reef.11.479-492 . hal-01381716

\section{HAL Id: hal-01381716 https://hal.science/hal-01381716}

Submitted on 14 Oct 2016

HAL is a multi-disciplinary open access archive for the deposit and dissemination of scientific research documents, whether they are published or not. The documents may come from teaching and research institutions in France or abroad, or from public or private research centers.
L'archive ouverte pluridisciplinaire HAL, est destinée au dépôt et à la diffusion de documents scientifiques de niveau recherche, publiés ou non, émanant des établissements d'enseignement et de recherche français ou étrangers, des laboratoires publics ou privés.

\section{(c)(1)}

Distributed under a Creative Commons Attribution| 4.0 International License 


\section{Instabilité et bifurcation du soufflage de membranes hyperélastiques}

\section{Nicolas Chevaugeon - Gilles Marckmann Erwan Verron - Bernard Peseux}

Laboratoire de Mécanique et Matériaux, Division Structures

École Centrale de Nantes

1 rue de la Nö̈, BP 92101

F-44321 Nantes Cedex 3

erwan.verron@ec-nantes.fr

RÉSUMÉ. Dans cet article, le comportement postbifurcatoire des membranes hyperélastiques soufflées est considéré. Les cas axisymétrique et tridimensionnel sont étudiés. Dans les deux cas le système algébrique obtenu est résolu en couplant l'algorithme de Newton-Raphson à la méthode de longueur d'arc. L'accent est mis sur l'étude des points singuliers et des branches secondaires. Finalement, quelques exemples numériques illustrent les développement effectués. ABSTRACT. This article deals with the post-bifurcating behaviour of inflated hyperelastic membranes. Axisymmetrical and three dimensional formulations are studied. In both cases, the resulting algebraic system is solved by the combining classical Newton-Raphson scheme and the arc-length continuation method. The emphasize is laid on singular points and secondary paths are pointed out. Finally, numerical examples are considered in order to illustrate the developments.

MOTS-CLÉS : membranes, hyperélasticité, soufflage, instabilité, bifurcation.

KEYWORDS: membranes, hyperelasticity, inflation, instability, bifurcation. 


\section{Introduction}

Les problèmes de membranes non-linéaires soufflées apparaissent dans différentes applications, par exemple en biomécanique [HSU 94] ou en mise en forme des plastiques [MAR 01]. La mise en équations de ces problèmes est basée sur les travaux précurseurs de Green et Adkins [GRE 60].

Dans le cas axisymétrique, le problème se réduit à un système d'équations différentielles avec des conditions limites aux deux extrémités de l'intervalle de définition, problème qui a été tout d'abord résolu par des méthodes couplant l'algorithme de Runge-Kutta avec la méthode du tir [YAN 70]. Plus récemment, les auteurs privilégient des approches numériques de type éléments finis [CHA 87, KHA 94]. Dans le cas général tridimensionnel, l'emploi de la méthode des éléments finis est systématique [VER 01b]

Différents auteurs ont mis en évidence la présence de phénomènes instables et de modes de bifurcation lors du soufflage de membranes élastiques non-linéaires. Expérimentalement, Alexander a isolé une configuration non-sphérique lors du soufflage d'un ballon sphérique en néoprène [ALE 71], et Kyriakides et Chang ont étudié l'apparition et la propagation d'une instabilité le long d'un tube en latex [KYR 91]. Ces problèmes d'instabilité ont été étudiés analytiquement et numériquement dans des cas simples : membranes sphériques [NEE 77, HAU 80] et cylindres finis [KHA 92]. En revanche, le comportement postbifurcatoire a été assez peu étudié. On peut cependant citer deux études axisymétriques utilisant la méthode des éléments finis : tout d'abord, celle de Duffet et Reddy [DUF 86] qui ont rencontré quelques difficultés pour suivre les branches secondaires et plus récemment, celle de Shi et Moita [SHI 96] qui ont utilisé une méthode de longueur d'arc et d'injection de mode pour explorer ces mêmes branches secondaires.

Dans le présent article, le comportement postbifurcatoire de membranes hyperélastiques soufflées est étudié. Dans le paragraphe suivant, les équations d'équilibre des membranes soumises à une pression extérieure ainsi que la loi de comportement adoptée sont rappelées. Ensuite, dans le troisième paragraphe, les méthodes numériques utilisées sont détaillées. Les méthodes de discrétisation axisymétrique et tridimensionnelle sont brièvement présentées. Puis, l'algorithme de résolution du système algébrique non-linéaire est précisé en mettant l'accent sur la méthode de continuation adoptée. Enfin, l'étude des points singuliers et la possibilité de calculer les branches secondaires de la courbe de chargement sont détaillées. Finalement, le paragraphe 4 présente plusieurs résultats numériques en mettant en évidence les instabilités obtenues par les approches axisymétrique et tridimensionnelle.

\section{Mise en équations des problèmes de soufflage de membranes}

\section{1. Équations d'équilibre. Principe des travaux virtuels}

On considère le soufflage quasistatique d'une membrane hyperélastique. Le matériau est supposé homogène, isotrope, élastique et incompressible. En tout point, l'épaisseur de la membrane est supposée beaucoup plus faible que le rayon de cour- 
bure, la géométrie de la membrane est donc décrite par la position de sa surface moyenne. La membrane est seulement soumise à une pression intérieure, $p$, et l'hypothèse des contraintes planes est faite.

Les configurations non-déformée et déformée sont respectivement notées $\mathcal{C}_{0}$ et $\mathcal{C}$. De façon similaire, les surfaces frontières sont respectivement $\partial \mathcal{C}_{0}$ et $\partial \mathcal{C}$. En considérant qu'il n'y a pas de force de volume et en négligeant les effets d'inertie, le principe des travaux virtuels s'écrit sous la forme suivante :

$$
r(\vec{u}, \overrightarrow{\delta u}, p)=\int_{\mathcal{C}_{0}} \delta W d V-\int_{\partial \mathcal{C}} \overrightarrow{\delta u} p \vec{n} d S=0 \quad \forall \overrightarrow{\delta u}
$$

Dans cette équation, $\delta \vec{u}$ est un déplacement virtuel cinématiquement admissible, $\vec{n}$ est la normale extérieure à la surface moyenne au point considéré et $W$ est l'énergie de déformation. Le premier terme intégral représente le travail virtuel des efforts intérieurs et le second terme intégral est le travail virtuel du chargement extérieur. Il convient de noter que ce second terme est défini sur la configuration déformée car la force due à la pression est une force suiveuse dont l'intensité dépend de la géométrie courante.

\subsection{Loi de comportement}

Dans cet article, les membranes étudiées sont supposées hyperélastiques et seul le modèle de Mooney-Rivlin est utilisé. La fonction énergie de déformation, $W$, est une fonction des deux premiers invariants, $I_{1}$ et $I_{2}$, du tenseur des dilatations de CauchyGreen droit, $\overline{\bar{C}}$ :

$$
W=C\left[\left(I_{1}-3\right)+\alpha\left(I_{2}-3\right)\right]
$$

où $C$ et $\alpha$ sont les deux constantes matérielles. Les deux premiers invariants de $\overline{\bar{C}}$ sont définis par:

$$
I_{1}=\operatorname{tr} \overline{\bar{C}}
$$

et :

$$
I_{2}=\frac{1}{2}\left[(t r \overline{\bar{C}})^{2}-\operatorname{tr}\left(\overline{\bar{C}}^{2}\right)\right]
$$

Le troisième invariant de $\overline{\bar{C}}, I_{3}=\operatorname{det} \overline{\bar{C}}$ est égal à 1 , de par l'hypothèse d'incompressibilité.

\section{Discrétisation et résolution numérique}

\subsection{Discrétisation}

\subsubsection{Cas axisymétrique}

Dans le cas axisymétrique, la membrane est réduite à une courbe continue. Chaque point matériel $P$ de cette courbe a pour coordonnées radiale $R(s)$ et axiale $Z(s)$ dans 
la configuration non déformée. $s$ représente la coordonnée curviligne le long de la courbe. La courbe est maintenant divisée en $n$ intervalles délimités par $n+1$ noeuds. Chacune des deux coordonnées est approchée par une spline cubique définie sur les noeuds. A l'instar des éléments finis isoparamétriques, on interpole les déplacements des points matériels sur la même base de fonctions B-splines. Pour plus de détails sur cette méthode d'interpolation, le lecteur peut se référer à [VER 01a].

\subsubsection{Cas tridimensionnel}

Dans le cas tridimensionnel, la discrétisation est effectuée à l'aide d'éléments finis classiques de type Q8.

Dans toute la suite, les deux cas seront traités simultanément, les systèmes discrétisés étant similaires. On note $\{U\}$ le vecteur des déplacements nodaux. Le Principe des Travaux Virtuels (1) devient :

$$
r(\vec{u}, \overrightarrow{\delta u}, p)=\{\delta U\}^{t}\{R(\{U\}, p)\}=0 \quad \forall\{\delta U\}^{t}
$$

où $\{R\}$ est le résidu du problème qui peut être écrit comme la différence entre les vecteurs nodaux des forces intérieures et des forces extérieures :

$$
\{R(\{U\}, p)\}=\left\{F_{\text {int }}(\{U\})\right\}-\left\{F_{\text {ext }}(\{U\}, p)\right\}=\{0\}
$$

\subsection{Méthode de résolution}

Le système précédent est bien évidemment non-linéaire. Sa résolution nécessite le calcul de la matrice raideur tangente $[K]$ définie par :

$$
[K]=\left[K_{i n t}\right]-\left[K_{e x t}\right]=\frac{\partial\left\{F_{i n t}\right\}}{\partial\{U\}}-\frac{\partial\left\{F_{\text {ext }}\right\}}{\partial\{U\}}
$$

De plus, il est bien connu que les problèmes de soufflage de membranes hyperélastiques présentent des points limites : dans le cas d'un ballon de baudruche, la courbe de soufflage volume du ballon-pression n'est pas monotone [BEA 87]. C'est pourquoi, l'obtention de la courbe d'équilibre nécessite l'emploi d'une méthode de continuation. Dans ce travail, nous utilisons la méthode de longueur d'arc, sous la forme simplifiée du contrôle en déplacement proposée par Batoz et Dhatt [BAT 79]. Ainsi, en supposant connu un point d'équilibre défini par le vecteur des déplacements nodaux $\left\{U_{e}\right\}$ et la pression $p_{e}$, le problème à résoudre consiste à déterminer les incréments de déplacement $\{\Delta U\}$ et de pression $\Delta p$ qui vérifient :

$$
\left\{\begin{array}{l}
\left\{R\left(\left\{U_{e}\right\}+\{\Delta U\}, p_{e}+\Delta p\right)\right\}=0 \\
\|\{\Delta U\}\|^{2}-d a^{2}=0
\end{array}\right.
$$

où la seconde équation est l'équation de longueur d'arc, $d a$ étant la longueur d'arc imposée. Ce système est résolu par l'algorithme itératif de Newton-Raphson. 
À chaque itération, on calcule les changements des incréments de déplacement et de pression, respectivement $\{d U\}$ et $d p$, en résolvant le système bordé :

$$
\left[\begin{array}{c|c}
{[K]} & -\frac{1}{p}\left\{F_{\text {ext }}\right\} \\
\hline 2\left\{\Delta U^{\text {prec }}\right\}^{t} & 0
\end{array}\right]\left\{\begin{array}{c}
\{d U\} \\
d p
\end{array}=-\left\{\frac{\left\{R \frac{\text { prec }}{}\right\}}{\left\|\Delta\left\{U^{\text {prec }}\right\}\right\|^{2}-d a^{2}}\right.\right.
$$

Dans cette équation, l'exposant $\star \frac{p r e c}{}$ représente la valeur de la grandeur $\star$ à l'itération précédente de l'algorithme de Newton-Raphson.

\subsection{Points singuliers et comportement postbifurcatoire}

Comme il a été mentionné précédemment, les courbes d'équilibre des membranes soufflées possèdent des points singuliers c'est-à-dire des points de la courbe d'équilibre pour lesquels le comportement change brusquement. Dans le cas du soufflage, on identifie deux types de points singuliers : les points limites pour lesquels la monotonie de la courbe de charge change et les points de bifurcation simple qui voient naître une nouvelle courbe d'équilibre solution du problème.

L'étude des points singuliers peut être divisée en trois étapes :

- détection de la présence d'un point singulier;

- isolation de ce point singulier;

- détermination de la nature du point singulier et dans le cas d'un point de bifurcation, exploration de la branche secondaire d'équilibre.

\subsubsection{Détection}

Pour détecter la présence des points singuliers, un critère inspiré des travaux de Sokol et Witkowski [SOK 97] est adopté. Ce critère permet de déterminer le point d'équilibre où la matrice raideur devient singulière. En notant la plus petite des valeurs propres négatives de en valeur absolue, le critère est le suivant :

- si le nombre des valeurs propres négatives de n'a pas changé depuis le dernier point d'équilibre :

- si ce nombre a changé :

Au point singulier, on a bien sûr :

\subsubsection{Isolation}

Pour isoler le point singulier, on utilise simplement la méthode de dichotomie sur la valeur de la longueur d'arc jusqu'à vérifier 


\subsubsection{Nature du point singulier}

En notant $\left\{Z_{\min }\right\}$ le vecteur propre correspondant à la valeur propre $w_{\min }$, le produit scalaire entre ce vecteur et le chargement extérieur permet de déterminer la nature du point singulier :

- si $\left\{Z_{\min }\right\}^{t} \cdot\left\{F_{\text {ext }}\right\}=0$ alors le point singulier est un point de bifurcation;

- dans le cas contraire, c'est un point limite.

\subsubsection{Passage sur une branche secondaire}

Dans le cas d'un point de bifurcation, le passage sur une branche secondaire nécessite seulement le calcul d'un point d'équilibre sur cette nouvelle branche. Pour cela, on utilise la méthode d'injection de mode propre utilisée avec succès par Shi et Moita [SHI 96]. Pour l'obtention de ce premier point d'équilibre sur la nouvelle branche, la phase de prédiction de l'algorithme de Newton-Raphson est effectuée en utilisant le vecteur :

$$
\left\{\Delta U_{\text {prdit }}\right\}=d a\left\{Z_{\min }\right\}
$$

La phase de correction permet alors de calculer un point d'équilibre sur la nouvelle branche. Par la suite, l'algorithme est utilisé de manière classique pour explorer cette nouvelle branche.

\section{Exemples}

Dans ce paragraphe, quelques exemples sont examinés en utilisant les formulations axisymétrique et tridimensionnelle, et en mettant en évidence les modes de bifurcation symétriques et asymétriques.

Tous les exemples qui suivent sont étudiés avec une membrane d'épaisseur initiale $H=0,01$ et de coefficients matériau $C=1$ et $\alpha \in\{0 ; 0,1 ; 0,25\} \cdot p$ désigne la pression interne et $\lambda=R / R_{0}$ l'extension principale radiale. La sphère et les cylindres ont un rayon initial $R_{0}=1$.

\subsection{Sphère}

Dans un premier temps nous validons nos modèles sur des cas connus analytiquement. La sphère gonflée présente l'avantage de rester sphérique au cours du chargement en pression dans le cas d'un matériau de type Mooney-Rivlin. Sa réponse est alors donnée par l'équation [VER 99] :

$$
-(--)(\quad)
$$




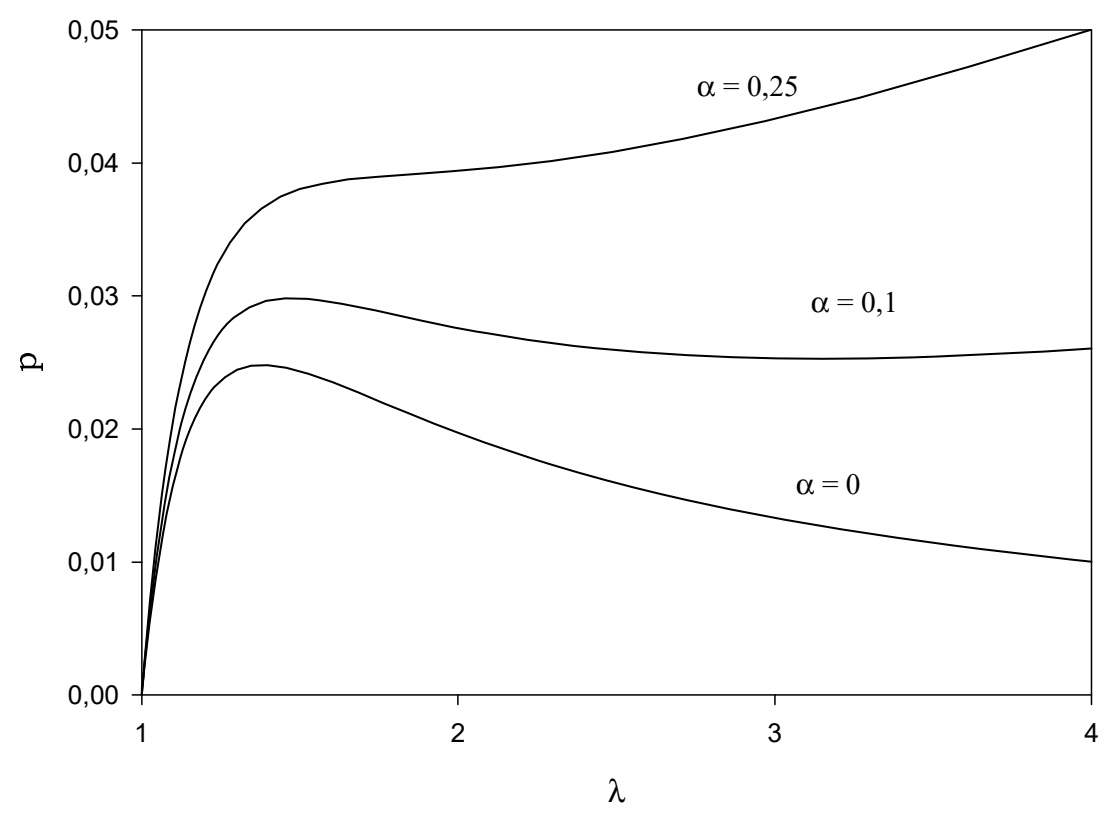

Figure 1. Solution du soufflage d'une membrane sphérique

Les résultats analytiques sont parfaitement retrouvés avec le modèle élément fini B-spline axisymétrique en utilisant 41 noeuds. Ces résultats numériques sont présentés sur la figure 1 pour trois valeurs du paramètre matériel $\alpha$.

Les points limites sont passés avec succès et aucun point de bifurcation n'est observé pour cette géométrie et ce type de matériau. Nous retrouvons ainsi les résultats de Haughton [HAU 80].

\subsection{Cylindre infini}

Un autre cas simple concerne la membrane cylindrique droite de longueur infinie. La réponse triviale, pour laquelle la déformée reste un cylindre droit, peut se réduire à l'équation suivante :

$$
p=\frac{2 C H}{R_{0}}(1+\alpha)\left(1-\frac{1}{\lambda^{4}}\right)
$$

Numériquement, nous modélisons un tronçon de cylindre de hauteur un élément fini B-spline axisymétrique à 41 noeuds. La réponse triviale du modèle vérifie bien l'équation (15) pour trois valeurs du paramètre matériel :

(figure 2),

(figure 3) et

(figure 4). Sur ces trois figures, la réponse triviale correspond aux courbes présentant une asymptote horizontale.

L'étude des points singuliers met en évidence des points de bifurcation fournissant des réponses non triviales sous la forme de lobes locaux déjà observés expérimentalement [KYR 91] et numériquement [SHI 96]. Les trois premiers points de bifurcation obtenus pour sont atteints pour des valeurs de pression égales à 
gure 2 donnant la pression interne $p$ en fonction du plus grand rayon de la membrane $R_{\max }\left(R_{\max }=R\right.$ pour la réponse triviale).

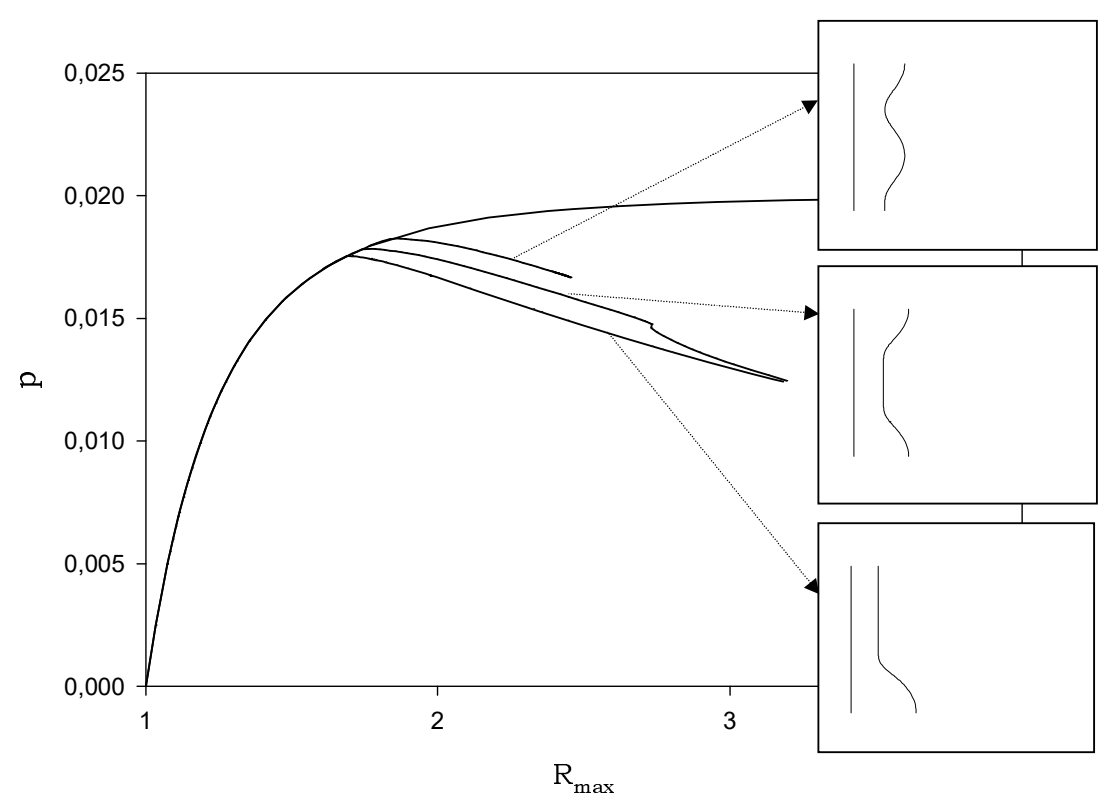

Figure 2. Solution d'une membrane cylindrique infinie : $\alpha=0$

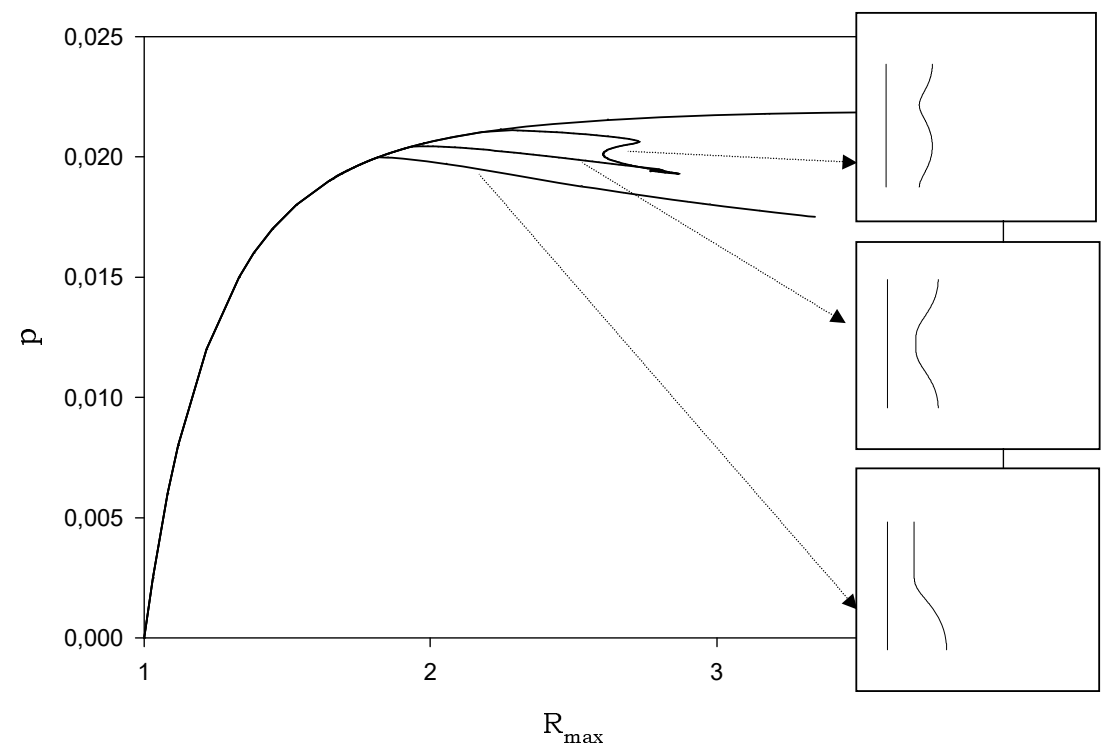

Figure 3. Solution d'une membrane cylindrique infinie : $\alpha=0,1$

De la même manière, trois modes de déformation similaires sont obtenus pour $\alpha=0,1$ (figure 3 ) pour des valeurs de pression valant respectivement 0,$0199 ; 0,0204$ et 0,0211 . Pour $\alpha=0,25$ (figure 4), seule la branche de bifurcation correspondant au premier mode de bifurcation relatif aux deux autres cas a pu être détectée. Ceci peut être dû à des problèmes de précision numérique lors des différents tests de l'analyse des points singuliers.

Dans les deux premiers cas, nous retrouvons bien les trois modes de bifurcation calculés par Shi et Moita [SHI 96] pour un matériau de type Ogden à trois termes. On peut cependant noter que les trois points de bifurcation correspondent à l'apparition respectivement de un, deux et trois lobes sur la longueur du tronçon de cylindre 
considéré. Nous pouvons en déduire, à la manière de Shi et Moita, que les trois premiers modes de bifurcation obtenus numériquement correspondront à des valeurs de pression différentes selon le rapport de forme $L_{0} / R_{0}$ du modèle de membrane infinie adopté. Ainsi, il existe une infinité de points de bifurcation pour ce problème.

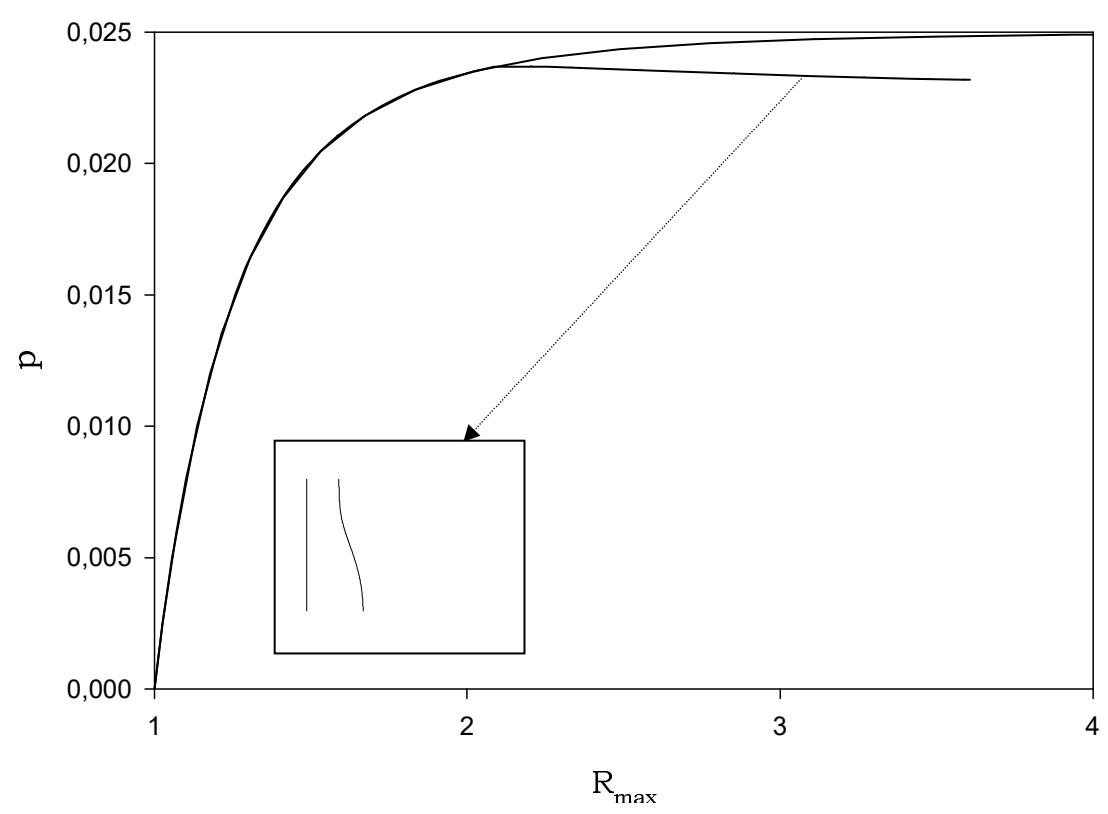

Figure 4. Solution d'une membrane cylindrique infinie : $\alpha=0,25$

\subsection{Cylindre encastré}

De la même manière que pour le cylindre infini, nous étudions le cylindre droit de même rapport de forme, encastré à ses deux extrémités. Contrairement au cas précédent, les modes de bifurcation ne correspondent pas à l'apparition de lobes, mais à la naissance d'une asymétrie de la déformée de la membrane par rapport au plan milieu. Il faut noter que la formation d'un pseudolobe (bulge en anglais) correspond à l'apparition d'un point limite caractérisé par un changement de signe de la pente de la courbe $p$ en fonction de $R_{\text {max }}$ et par le passage vers une configuration instable, et ne représente pas un mode de bifurcation.

Dans le cas d'un matériau néo-hookéen $(\alpha=0)$, le point limite apparait pour $p=0,0169$, puis des points de bifurcation apparaissent pour des valeurs de $p$ de 0,$0153 ; 0,0128$ et 0,01102 . Les courbes $p\left(R_{\max }\right)$ sont très proches les unes des autres. La figure 5 illustre les différentes configurations obtenues.

Pour une valeur du coefficient matériel $\alpha=0,1$; les mêmes phénomènes sont observés avec un pseudo-lobe moins marqué. Le premier point limite correspond à $p=0,0195$, tandis que les points de bifurcation sont obtenus pour $p=0,0194$ (très proche du point limite) pour le premier mode et deux autres points correspondant à $p=0,0175$. Ces deux points conduisent à deux modes de bifurcation symétriques par rapport au plan de mi-hauteur du cylindre. Finalement, un second point limite correspondant à un nouveau changement de signe de la pente de la courbe de charge est 
observé pour une pression de 0,0162 . La figure 6 illustre les différentes configurations obtenues.

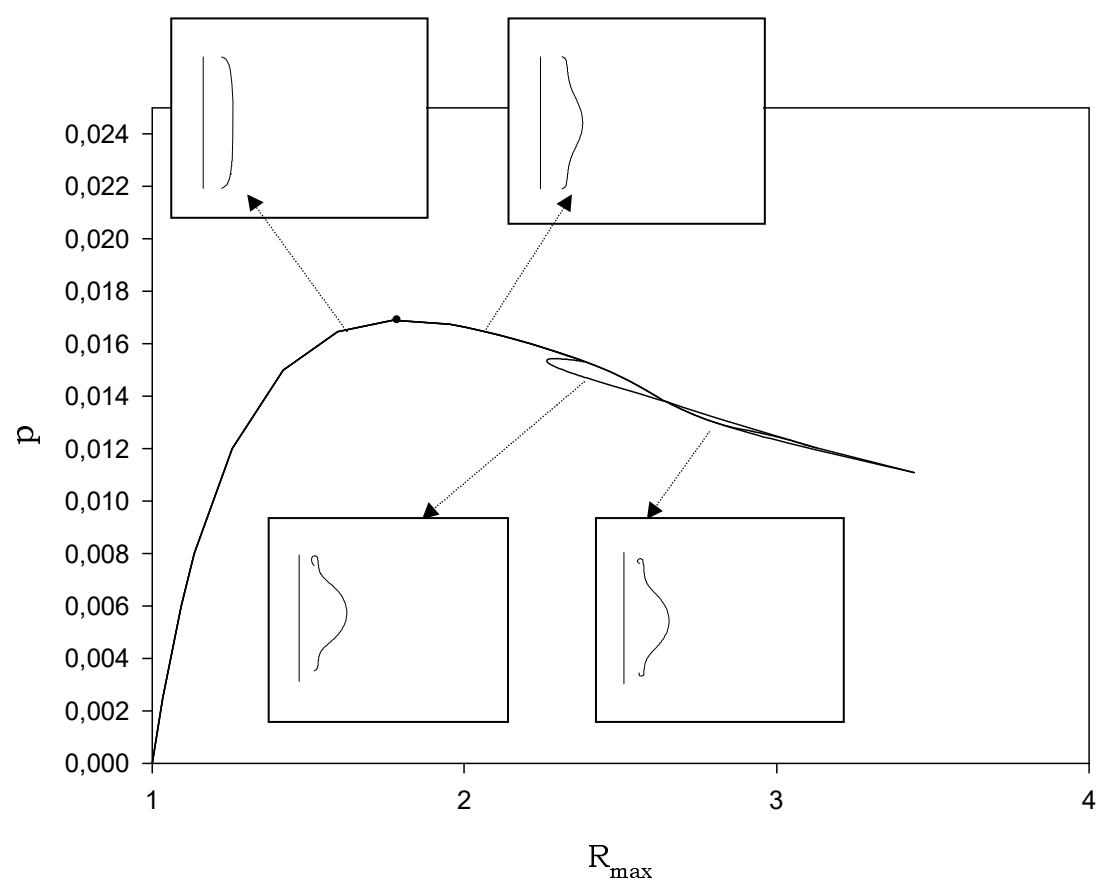

Figure 5. Solution d'une membrane cylindrique encastrée :

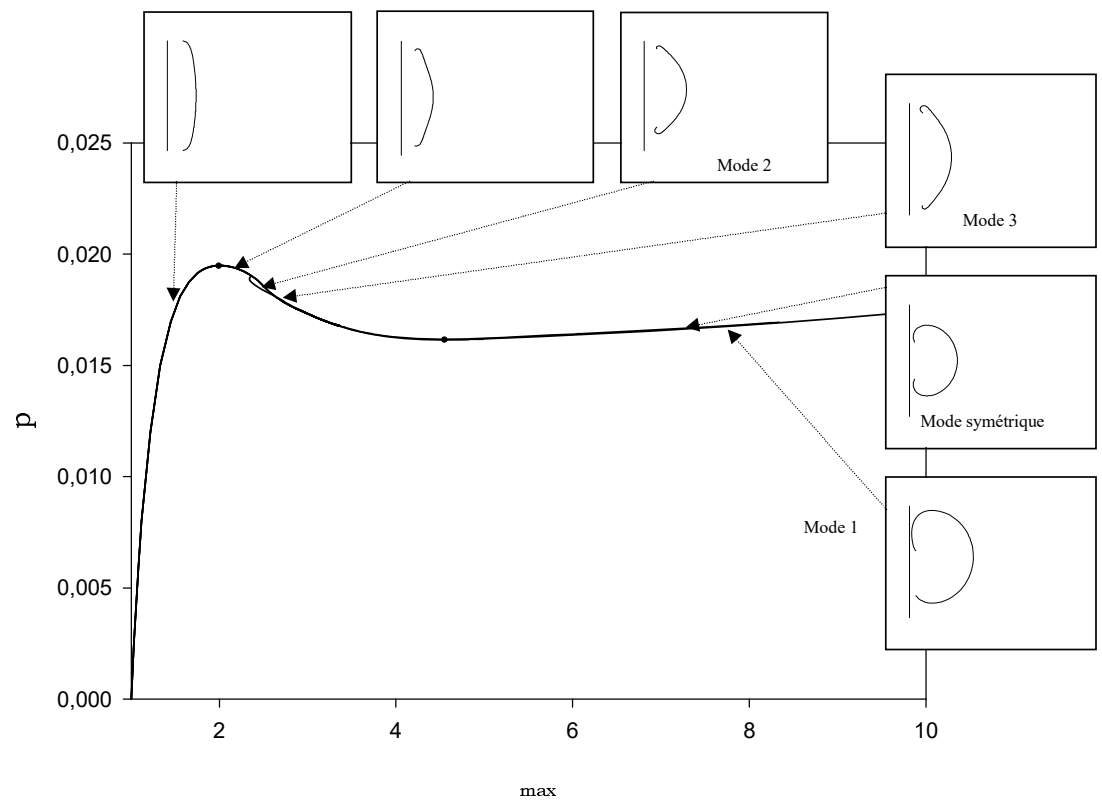

Figure 6. Solution d'une membrane cylindrique encastrée :

Pour , deux points limites apparaissent également, pour des valeurs de pression égales à et . Un seul point de bifurcation a pu être détecté, comme dans le cas du cylindre de longueur infinie.

Ce mode de bifurcation est obtenu pour , juste avant le deuxième point limite. Il correspond à nouveau à un mode asymétrique. Dans le cas présent, la branche symétrique et la branche secondaire asymétrique sont pratiquement confondues comme le montre la figure 7. Seule l'observation de la déformée permet de distinguer les deux cas. 


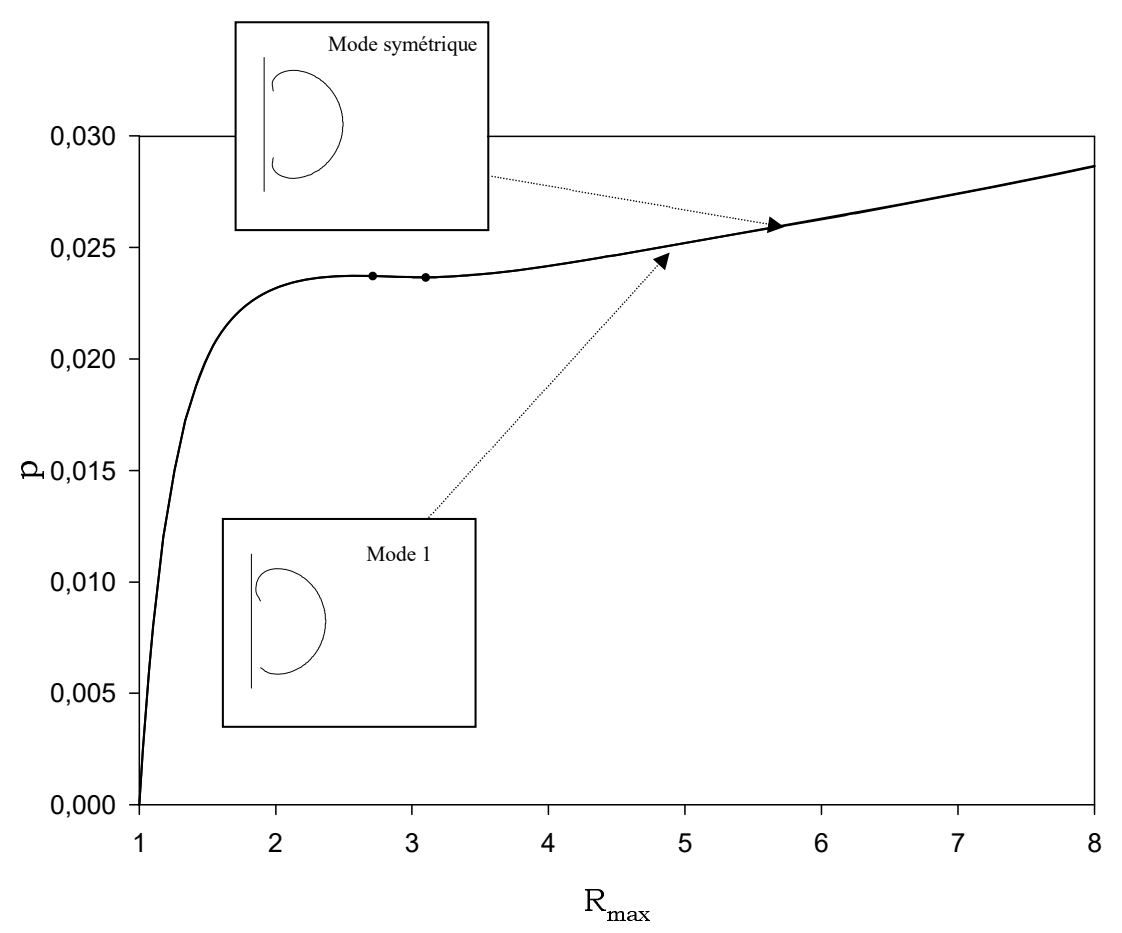

Figure 7. Solution d'une membrane cylindrique encastrée : $\alpha=0,25$

\subsection{Tore}

Nous nous intéressons maintenant au cas d'un tore de rayon moyen initial $R_{\text {moyen }}=$ 40 ayant une section circulaire de rayon $R_{s}=10$.

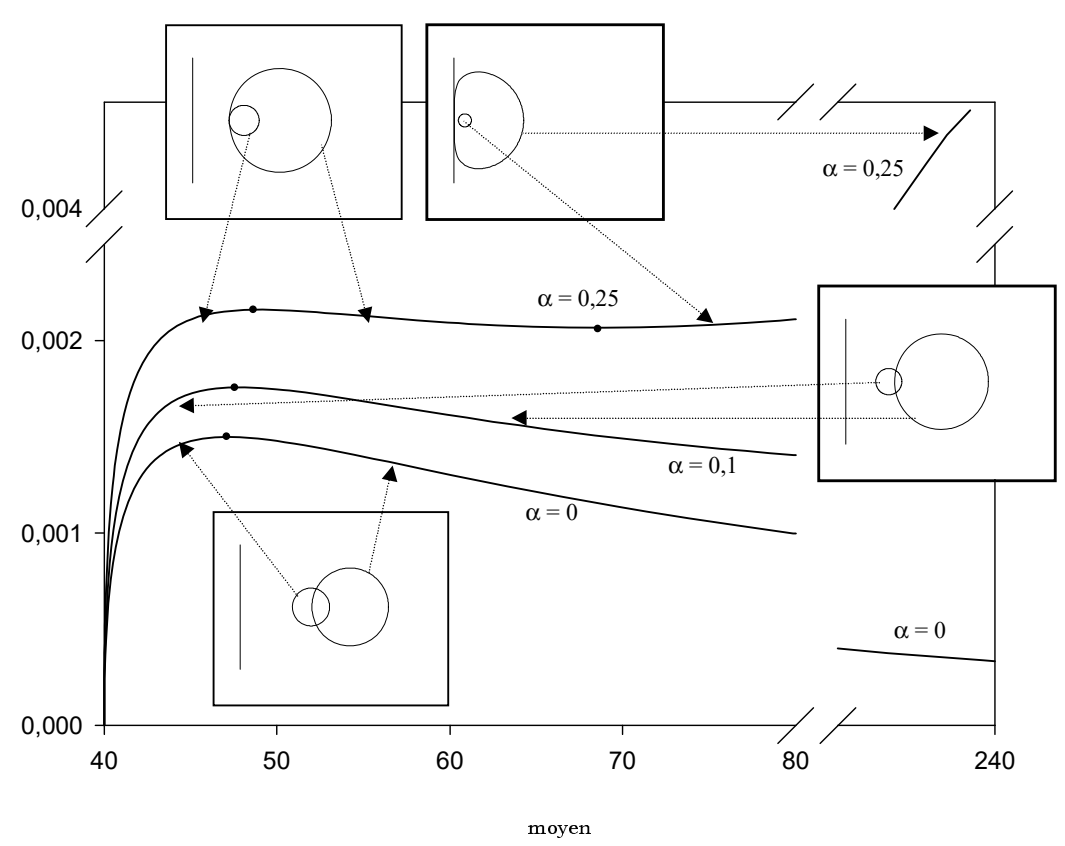

Figure 8. Solution d'une membrane torique

Tout d'abord, il faut noter que la modélisation axisymétrique ne met en évidence aucun point singulier de type bifurcation. Cependant la présence de points limites est mise en évidence. Ils correspondent à $p=0,0015$ lorsque $\alpha=0, p=0,00176$ lorsque $\alpha=0,1$, et à $p=0,00216$ et $p=0,00207$ lorsque $\alpha=0,25$. De plus, 
on observe que la section du tore reste quasiment circulaire lors du soufflage pour les valeurs matérielles $\alpha=0$ et $\alpha=0,1$. Tous ces résultats sont résumés sur la figure 8 .

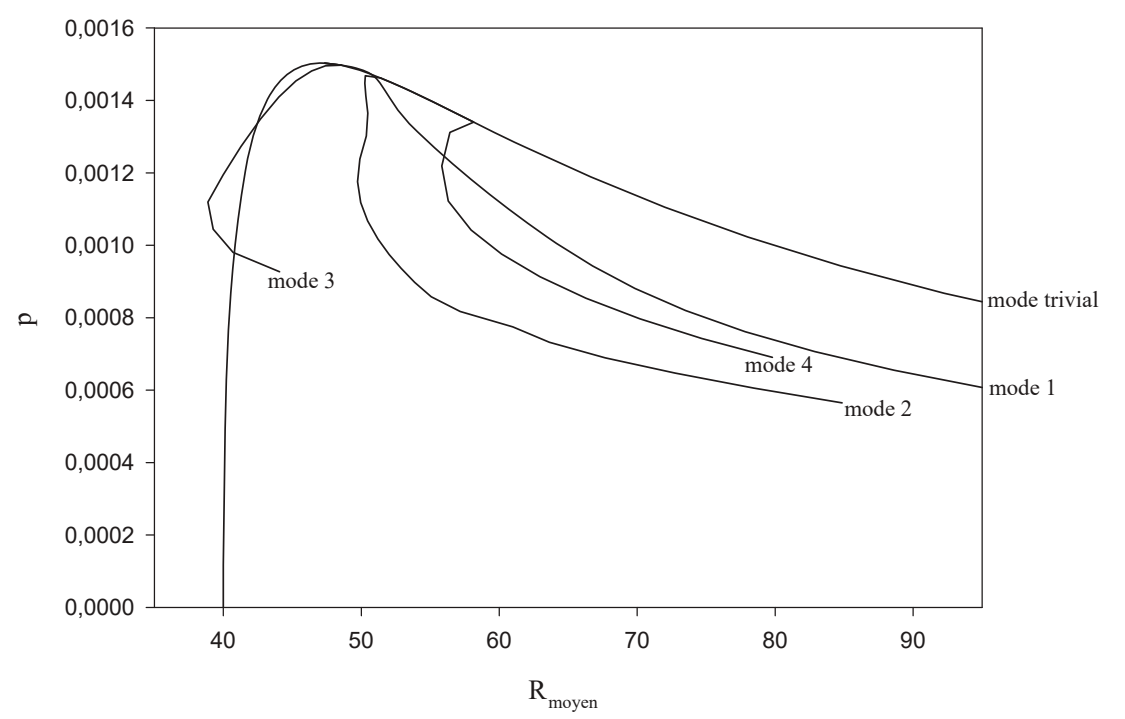

Figure 9. Courbes de charge d'une membrane torique

Dans le cas de la simulation tridimensionnelle, des modes de bifurcation sont mis en évidence. Ceci confirme les résultats obtenus par Reese et Wriggers pour un matériau de type Ogden [REE 95] : dans le cas d'une membrane torique, il n'y a que des modes asymétriques. Les figures 9 et 10 présentent respectivement les différentes branches obtenues et la forme des modes correspondants.
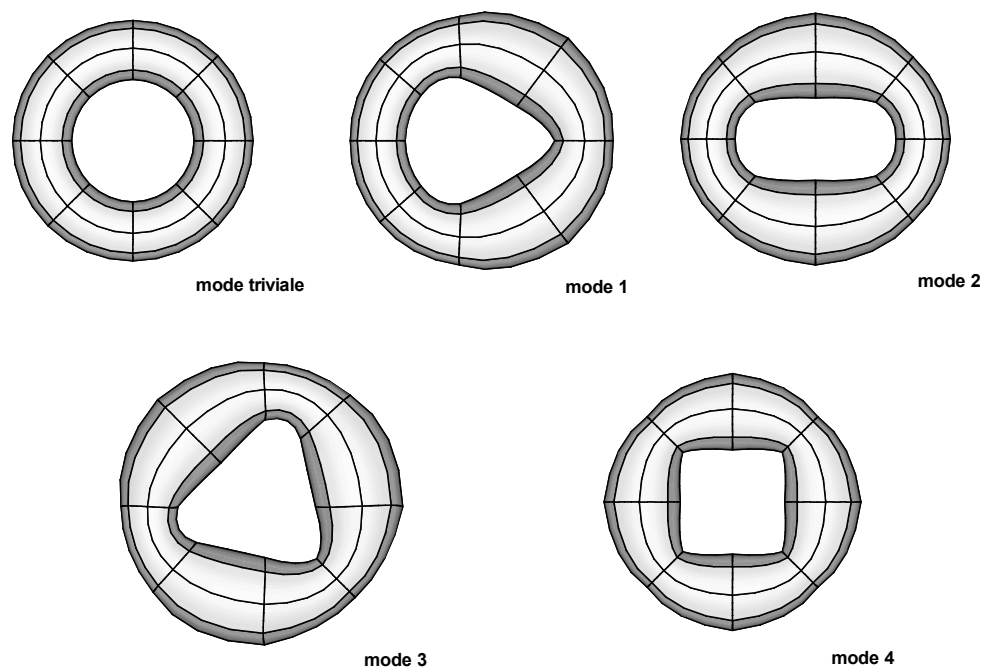

Figure 10. Modes de bifurcation d'une membrane torique

\section{Conclusion}

Dans cette étude, le comportement postbifurcation des membranes hyperélastiques soumises à des chargements en pression a été étudié par des approches numériques 
axisymétrique et tridimensionnelle. Le critère permettant la détection des points singuliers portant sur le signe des valeurs propres de la matrice raideur s'est avéré très efficace. La qualité de la méthode numérique consistant à utiliser l'injection du mode propre correspondant à la valeur propre nulle du système pour explorer les branches secondaires a été mise en évidence. Les différents exemples traités montrent l'importance des modes de bifurcation asymétriques dans le comportement des structures de type membranes. Des travaux sont actuellement en cours pour améliorer la modélisation des membranes tridimensionnelles en grandes déformations pour permettre la construction de modèles plus réduits permettant de concentrer le temps de calcul sur l'étude des points singuliers.

\section{Bibliographie}

[ALE 71] Alexander H., " The tensile instability of initially spherical balloons », Int. J. Engng Sci., vol. 9, 1971, p. 151-162.

[BAT 79] BATOZ J. L., DhATT G., « Incremental displacement algorithms for nonlinear problems », Int. J. Num. Meth. Engng, vol. 14, 1979, p. 1262-1267.

[BEA 87] BEATTY M. F., « Topics in finite elasticity : hyperelasticity of rubber, elastomers, and biological tissues - with examples ", Appl. Mech. Rev., vol. 40, n 12, 1987, p. 16991734.

[CHA 87] Charrier J. M., Shrivastava S., Wu R., « Free and constrained inflation of elastic membranes in relation to thermoforming - Axisymmetric problems », J. Strain Analysis, vol. 22, $\mathrm{n}^{\circ} 2,1987$, p. 115-125.

[DUF 86] Duffet G. A., Reddy B. D., « The solution of multi-parameter systems of equations with application to problems in nonlinear elasticity », Comp. Meth. Appl. Mech. Engng, vol. 59, 1986, p. 179-213.

[GRE 60] Green A. E., Adkins J. E., Large elastic deformations, The Clarendon Press, Oxford, 1960.

[HAU 80] HaUghton D. M., " Post-bifurcation of perfect and imperfect spherical elastic membranes », Int. J. Solids Structures, vol. 16, 1980, p. 1123-1133.

[HSU 94] Hsu F. P. K., Schwab C., Rigamonti D., Humphrey J. D., «Identification of response functions from axisymmetric membrane inflation tests : implications for biomechanics », Int. J. Solids Struct., vol. 31, n 24, 1994, p. 3375-3386.

[KHA 92] Khayat R. E., Derdouri A., GARCIA-RÉJON A., «Inflation of an elastic cylindrical membrane : non-linear deformation and instability », Int. J. Solids Structures, vol. $29, \mathrm{n}^{\circ} 1,1992$, p. 69-87.

[KHA 94] KHAYAT R. E., DERDOURI A., «Inflation of hyperelastic cylindrical membranes as applied to blow moulding. part I. axisymmetric case », Int. J. Num. Meth. Eng., vol. 37, 1994, p. 3773-3791.

[KYR 91] KYRIAKIDES S., CHANG Y., " The initiation and propagation of a localized instability in an inflated elastic tube », Int. J. Solids Struct., vol. 27, n 9, 1991, p. 1085-1111.

[MAR 01] Marckmann G., Verron E., Peseux B., « Finite element analysis of blow molding and thermoforming using a dynamic explicit procedure », Polym. Eng. Sci., vol. $41, n^{\circ} 3,2001$, p. $426-439$. 
[NEE 77] NeEdLEman A., «Inflation of spherical rubber balloons », Int. J. Solids Structures, vol. 13,1977, p. 409-421.

[REE 95] REESE S., Wriggers P., « A finite element method for stability problems in finite elasticity », Int. J. Num. Meth. Eng., vol. 38, 1995, p. 1171-1200.

[SHI 96] SHi J., MOITA G. F., « The post-critical analysis of axisymmetric hyper-elastic membranes by the finite element method », Comput. Methods Appl. Mech. Engng, vol. 135, 1996, p. 265-281.

[SOK 97] SokóL T., WiTKOWSKi M., "Some experiences in the equilibrium path determination », Comput. Assist. Mech. Engng Sci., vol. 4, 1997, p. 189-208.

[VER 99] Verron E., Khayat R. E., A. A. D., Peseux B., « Dynamic inflation of hyperelastic spherical membranes », J. Rheol., vol. 43, nº 5, 1999, p. 1083-1097.

[VER 01a] VERron E., MARCKMANN G., « An axisymmetric B-spline model for the nonlinear inflation of rubberlike membranes », Comput. Meth. Appl. Mech. Engng, vol. 190, $n^{\circ}$ 46-47, 2001, p. 6271-6289.

[VER 01b] Verron E., Marckmann G., Peseux B., " Dynamic inflation of non-linear elastic and viscoelastic rubberlike membranes », Int. J. Num. Meth. Engng, vol. 50, n ${ }^{\circ}$, 2001, p. 1233-1251.

[YAN 70] YANG W. H., FENG W. W., « On axisymmetrical deformations of nonlinear membranes », J. Appl. Mech. ASME, vol. 37, 1970, p. 1002-1011. 